PHYSICAL REVIEW E 82, 046111 (2010)

\title{
Generalized centrifugal-force model for pedestrian dynamics
}

\author{
Mohcine Chraibi* $^{*}$ and Armin Seyfried ${ }^{\dagger}$ \\ Jülich Supercomputing Centre, Forschungszentrum Jülich, 52425 Jülich, Germany \\ Andreas Schadschneider \\ Institute for Theoretical Physics, Universität zu Köln, D-50937 Köln, Germany
}

(Received 25 August 2010; published 20 October 2010)

\begin{abstract}
A spatially continuous force-based model for simulating pedestrian dynamics is introduced which includes an elliptical volume exclusion of pedestrians. We discuss the phenomena of oscillations and overlapping which occur for certain choices of the forces. The main intention of this work is the quantitative description of pedestrian movement in several geometries. Measurements of the fundamental diagram in narrow and wide corridors are performed. The results of the proposed model show good agreement with empirical data obtained in controlled experiments.
\end{abstract}

DOI: 10.1103/PhysRevE.82.046111

PACS number(s): 89.40.-a, 45.70.Vn, 34.10.+x

\section{INTRODUCTION}

For a beneficial application of pedestrians dynamics, robust and quantitatively verified models are required. A wide spectrum of models has been designed to simulate pedestrian dynamics. Generally these models can be classified into macroscopic and microscopic models. In macroscopic models the system is described by mean values of characteristics of pedestrian streams, e.g., density and velocity, whereas microscopic models consider the movement of individual persons separately. Microscopic models can be subdivided into several classes e.g., rule-based and force-based models. For a detailed discussion, we refer to $[1,2]$. In this work we focus on spatially continuous force-based models.

Force-based models take Newton's second law of dynamics as a guiding principle. Given a pedestrian $i$ with coordinates $\vec{R}_{i}$ we define the set of all pedestrians that influence pedestrian $i$ at a certain moment as

$$
\mathcal{N}_{i}:=\left\{j:\left\|\vec{R}_{j}-\vec{R}_{i}\right\| \leq r_{c} \wedge i \text { “ feels } " j\right\},
$$

where $r_{c}$ is a cutoff radius. We say pedestrian $i$ "feels" pedestrian $j$ if the line joining their centers of mass does not intersect any obstacle. In a similar way we define the set of walls or borders that act on pedestrian $i$ as

$$
\mathcal{W}_{i}:=\left\{w:\left\|\vec{R}_{w_{i}}-\vec{R}_{i}\right\| \leq r_{c}\right\}
$$

where $w_{i} \in w$ is the nearest point on the wall $w$ to the pedestrian $i$.

Thus, the movement of each pedestrian is defined by the equation of motion

\footnotetext{
*m.chraibi@fz-juelich.de

†a.seyfried@fz-juelich.de

†as@thp.uni-koeln.de
}

$$
m_{i} \ddot{\vec{R}_{i}}=\vec{F}_{i}=F_{i}^{\mathrm{drv}}+\sum_{j \in \mathcal{N}_{i}} F_{i j}^{\overrightarrow{\mathrm{eep}}}+\sum_{w \in \mathcal{W}_{i}} F_{i w}^{\overrightarrow{\mathrm{eep}}},
$$

where $F_{i j}^{\overrightarrow{\mathrm{eep}}}$ denotes the repulsive force from pedestrian $j$ acting on pedestrian $i, F_{i w}^{\overrightarrow{\text { rep }}}$ is the repulsive force emerging from the obstacle $w$ and $F_{i}^{\text {drv }}$ is a driving force. $m_{i}$ is the mass of pedestrian $i$.

The repulsive forces model the collision-avoidance performed by pedestrians and should guarantee a certain volume exclusion for each pedestrian. The driving force, on the other hand, models the intention of a pedestrian to move to some destination and walk with a certain desired speed. The set of Eqs. (3) for all pedestrians results in a high-dimensional system of second order ordinary differential equations. The time evolution of the positions and velocities of all pedestrians is obtained by numerical integration.

Most force-based models describe the movement of pedestrians qualitatively well. Collective phenomena like lane formation [3-5], oscillations at bottlenecks [3,4], the "fasteris-slower" effect $[6,7]$, clogging at exit doors $[4,5]$ are reproduced. These achievements indicate that these models are promising candidates for realistic simulations. However, a qualitative description is not sufficient if reliable statements about critical processes, e.g., emergency egress, are required. Moreover, implementations of models often require additional elements to guarantee realistic behavior, especially in high density situations. Here strong overlapping of pedestrians $[5,6]$ or negative and high velocities $[3,8]$ occur which then has to be rectified by replacing the equation of motion (3) by other procedures.

Force-based models contain free parameters that can be adequately calibrated to achieve a good quantitative description [9-13]. However, depending on the simulated geometry the set of parameters often changes. In most works quantitative investigations of pedestrian dynamics were restricted to a specific scenario or geometry, like one-dimensional motion [14], behavior at bottlenecks $[11,19,20]$, two-dimensional motion [12] or outflow from a room [15-18].

In this work, we restrict ourselves to corridors and address the possibility of describing the movement of pedestrians in 


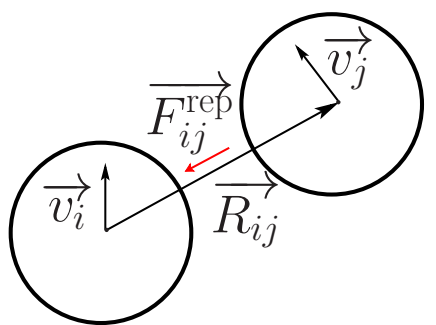

FIG. 1. (Color online) Direction of the repulsive force.

wide and narrow corridors reasonably and in a quantitative manner with a unique set of parameters. At the same time, the modeling approach should be as simple as possible.

In the next section, we propose such a model which is solely based on the equation of motion (3). Furthermore the model incorporates free parameters which allow calibration to fit quantitative data.

\section{CENTRIFUGAL FORCE MODEL}

The centrifugal force model (CFM) [5] takes into account the distance between pedestrians as well as their relative velocities. Pedestrians are modeled as circular disks with constant radius. Their movement is a direct result of superposition of repulsive and driving forces acting on the center of each pedestrian. Repulsive forces acting on pedestrian $i$ from other pedestrians in their neighborhood and eventually from, e.g., walls and stairs to prevent collisions and overlapping. The driving force, however, adds a positive term to the resulting force, to enable movement of pedestrian $i$ in a certain direction with a given desired speed $v_{i}^{0}$. The mathematical expression for the driving force is given by

$$
F_{i}^{\mathrm{drv}}=m_{i} \frac{\overrightarrow{v_{i}^{0}}-\overrightarrow{v_{i}}}{\tau}
$$

with a time constant $\tau$.

Given the direction connecting the positions of pedestrians $i$ and $j$,

$$
\overrightarrow{R_{i j}}=\overrightarrow{R_{j}}-\overrightarrow{R_{i}}, \quad \overrightarrow{e_{i j}}=\frac{\overrightarrow{R_{i j}}}{R_{i j}}
$$

The repulsive force then reads (see Fig. 1)

$$
F_{i j}^{\overrightarrow{\mathrm{rep}}}=-m_{i} k_{i j} \frac{v_{i j}^{2}}{R_{i j}} \overrightarrow{e_{i j}} .
$$

This definition of the repulsive force in the CFM reflects several aspects. First, the force between two pedestrians decreases with increasing distance. In the CFM it is inversely proportional to their distance $R_{i j}$. Furthermore, the repulsive force takes into account the relative velocity $v_{i j}$ between pedestrian $i$ and pedestrian $j$. The following special definition provides that slower pedestrians are not affected by the presence of faster pedestrians in front of them,

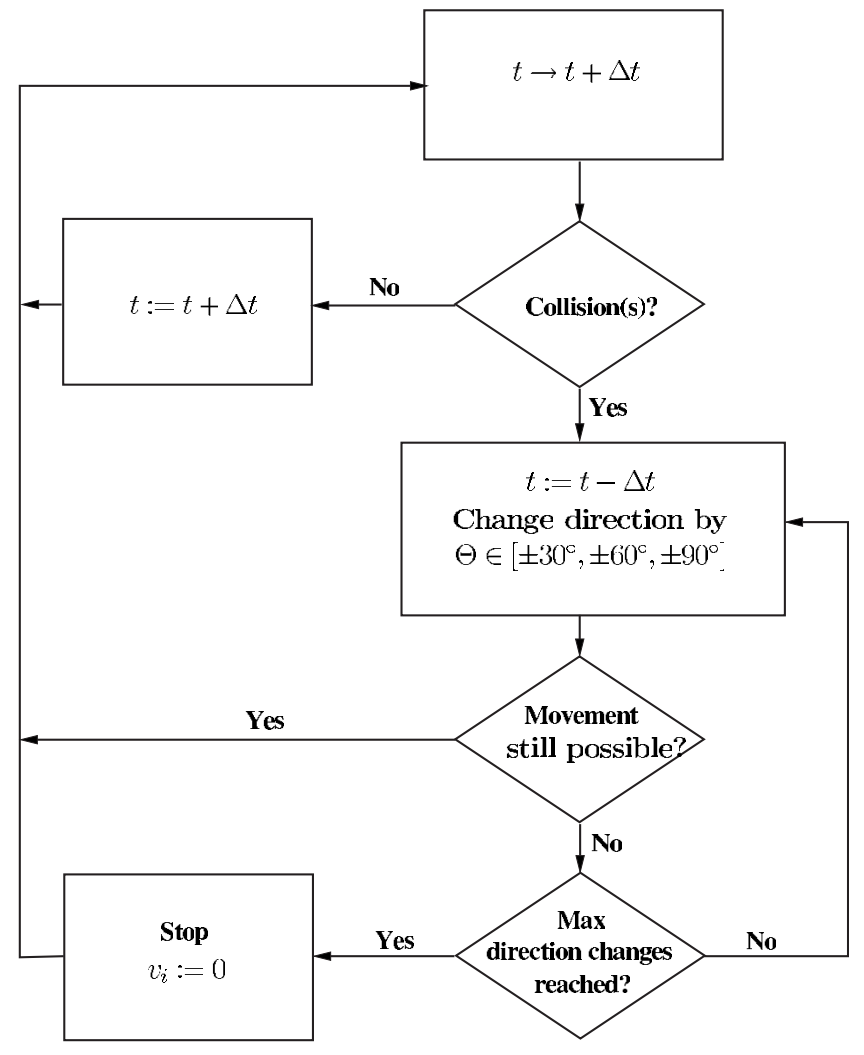

FIG. 2. Schematic representation of the collision detection technique (CDT), which is an important component in the CFM [5], to manage collisions and mitigate overlapping among pedestrians. In our model we do not need the CDT, which is a considerable simplification in comparison to the CFM [5].

$$
\begin{aligned}
v_{i j} & =\frac{1}{2}\left[\left(\overrightarrow{v_{i}}-\overrightarrow{v_{j}}\right) \cdot \overrightarrow{e_{i j}}+\left|\left(\overrightarrow{v_{i}}-\overrightarrow{v_{j}}\right) \cdot \overrightarrow{e_{i j}}\right|\right] \\
& =\left\{\begin{array}{cc}
\left(\overrightarrow{v_{i}}-\overrightarrow{v_{j}}\right) \cdot \overrightarrow{e_{i j}} & \text { if } \\
0 & \left(\overrightarrow{v_{i}}-\overrightarrow{v_{j}}\right) \cdot \overrightarrow{e_{i j}}>0
\end{array}\right.
\end{aligned}
$$

As in general pedestrians react only to obstacles and pedestrians that are within their perception, the reaction field of the repulsive force is reduced to the angle of vision $\left(180^{\circ}\right)$ of each pedestrian, by introducing the coefficient

$$
\begin{aligned}
k_{i j} & =\frac{1}{2} \frac{\overrightarrow{v_{i}} \cdot \overrightarrow{e_{i j}}+\left|\overrightarrow{v_{i}} \cdot \overrightarrow{e_{i j}}\right|}{v_{i}} \\
& =\left\{\begin{array}{cc}
\left(\overrightarrow{v_{i}} \cdot \overrightarrow{e_{i j}}\right) / v_{i} & \text { if } \overrightarrow{v_{i}} \cdot \overrightarrow{e_{i j}}>0 \quad \& \quad v_{i} \neq 0 \\
0 & \text { otherwise. }
\end{array}\right.
\end{aligned}
$$

The coefficient $k_{i j}$ is maximal when pedestrian $j$ is in the direction of movement of pedestrian $i$ and minimal when the angle between $j$ and $i$ is bigger than $90^{\circ}$. Thus the strength of the repulsive force depends on the angle.

As mentioned earlier the CFM is complemented with a "collision detection technique" (CDT) to manage conflicts and mitigate overlappings between pedestrians. Figure 2 depicts schematically the definition of the CDT. 
Although CDT is relatively simple, it adds an amount of complexity to the initial model defined with Eq. (3) and masks the main idea behind the repulsive forces. In the following we systematically modify the expression of the repulsive force to enable a better quantitative description of pedestrian dynamics.

\section{OVERLAPPING VS OSCILLATION}

In this work, we consider a velocity-dependent volume exclusion of pedestrians. Overlapping between two pedestrians occurs when their geometrical form (circle, ellipse,...) overlaps. Modeling a pedestrian as a circle or ellipse is just an approximation of the human body. Therefore, a certain amount of overlapping could be acceptable and might be interpreted as "elastic deformation." However, for the deformed body the center of mass no longer coincides with the center of the circle or ellipse. For this reason overlapping is a serious problem that should be dealt with.

In [21], it was shown that the introduction of a CDT is necessary to mitigate overlapping among pedestrians. The CDT keeps pedestrians away from each other with a distance of at least $r$, where $r$ represents the radius of the circle modeling the volume exclusion of pedestrians.

Our goal is to simplify the model by dispensing with the CDT and improve the repulsive force to compensate for the effects of the missing CDT on the dynamics. To introduce the shape of the modeled pedestrians in Eq. (6) we transform the singularity of the repulsive force from 0 to $2 r$,

$$
\overrightarrow{F_{i j}^{\mathrm{rep}}}=-m_{i} k_{i j} \frac{v_{i j}^{2}}{R_{i j}-2 r} \overrightarrow{e_{i j}} .
$$

Due to the quotient in Eq. (9) when the distance is small, low relative velocities lead to an unacceptably small force. Consequently, partial or total overlapping is not prevented. Introducing the intended speed in the numerator of the repulsive force eliminates this side-effect. This dependence on the desired speed is motivated by the observation that for faster pedestrians stronger repulsive forces are required to avoid collisions with other pedestrians and obstacles. Thus, the repulsive force is changed to

$$
F_{i j}^{\overrightarrow{\mathrm{rep}}}=-m_{i} k_{i j} \frac{\left(\eta v_{i}^{0}+v_{i j}\right)^{2}}{R_{i j}-2 r} \overrightarrow{e_{i j}},
$$

with a free parameter $\eta$ to adjust the strength of the force.

Those two changes in the repulsive force cause the emergence of two phenomena: Overlapping and oscillations. In the following we will define quantities to study those phenomena.

Avoiding overlapping between pedestrians and oscillations in their trajectories is difficult to accomplish in forcebased models. On one hand, increasing the strength of the repulsive force with the aim of excluding overlapping during simulations leads to oscillations in the trajectories of pedestrians. Consequently backward movements occur, which is not realistic especially in evacuation scenarios.

On the other hand, reducing the strength of the repulsive force (to avoid oscillations) leads inevitably to overlapping

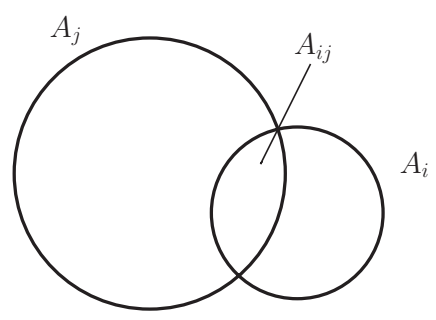

FIG. 3. The overlapping area between pedestrians $i$ and $j$ varies between 0 and 1 .

between pedestrians or between pedestrians and obstacles.

To solve this dilemma one has to find an adequate value of the strength of the repulsive force: it should neither be too high so that oscillations will appear, nor too low so that overlapping will be observed.

To understand this duality we quantify overlapping and oscillations during simulations. First, we define an overlapping-proportion during a simulation as

$$
o^{(v)}=\frac{1}{n_{o v}} \sum_{t=0}^{t=t_{\text {end }}} \sum_{i=1}^{i=N} \sum_{j>i}^{j=N} o_{i j},
$$

with

$$
o_{i j}=\frac{A_{i j}}{\min \left(A_{i}, A_{j}\right)} \leq 1,
$$

where $N$ is the number of simulated pedestrians. $A_{i j}$ is the overlapping area of the circles $i$ and $j$ with areas $A_{i}$ and $A_{j}$, respectively (see Fig. 3). $n_{o v}$ is the cardinality of the set

$$
\mathcal{O}:=\left\{o_{i j}: o_{i j} \neq 0\right\} \text {. }
$$

For $n_{o v}=0, o^{(v)}$ is set to zero.

For a pedestrian with velocity $\overrightarrow{v_{i}}$ and desired velocity $\overrightarrow{v_{i}^{0}}$ we define the oscillation-proportion as

$$
o^{(s)}=\frac{1}{n_{o s}} \sum_{t=0}^{t=t_{\text {end }}} \sum_{i=1}^{i=N} S_{i},
$$

where $S_{i}$ quantifies the oscillation-strength of pedestrian $i$ and is defined as follows:

$$
S_{i}=\frac{1}{2}\left(-s_{i}+\left|s_{i}\right|\right)
$$

with

$$
s_{i}=\frac{{\overrightarrow{v_{i}}}_{v_{i}}}{\left(v_{i}^{0}\right)^{2}},
$$

and $n_{o s}$ is the cardinality of the set

$$
\mathcal{S}:=\left\{s_{i}: s_{i} \neq 0\right\} .
$$

Here again $o^{(s)}$ is set to zero if $n_{o s}=0$. The proportions $o^{(v)}$ and $o^{(s)}$ are normalized to 1 and describe the evolution of the phenomena overlapping and oscillations during a simulation.

In order to exemplify the behavior of these two coupled phenomena we simulate an evacuation of 35 pedestrian from a $4 \times 4 \mathrm{~m}^{2}$ room with an exit of $1.2 \mathrm{~m}$ and determine $o^{(v)}$ 


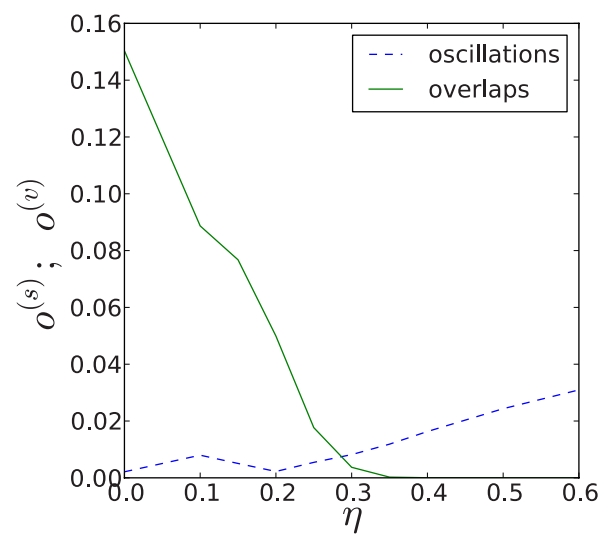

FIG. 4. (Color online) Oscillation-proportion $o^{(s)}$ and overlapping-proportion $o^{(v)}$ as function of the interaction strength $\eta$ obtained from 200 simulations with different initial conditions. Oscillations increase with increasing strength of the repulsive force, while overlaps become negligible for larger $\eta$. The case $\eta=0$ is the CFM. In each run the simulations for different $\eta$ are started with the same initial values.

and $o^{(s)}$ for different values of $\eta$ in Eq. (10). Results are shown in Fig. 4. $\eta=0$ is a special case of the model and represents the CFM [5]. The high values of the overlapping proportion suggest that simulations using only CFM without the CDT lead to unreasonable results. For further details we refer to [21].

The introduction of the intended velocity in the repulsive force enhances the ability of the repulsive force to guarantee the volume exclusion of pedestrians. This is reflected by the decreasing of the overlapping-proportion $o^{(v)}$ while increasing $\eta$ [Eq. (10)]. See Fig. 4.

Meanwhile, the oscillation-proportion $o^{(s)}$ increases, thus the system tends to become instable. Large values of the oscillation-proportion $o^{(s)}$ imply less stability. For $s_{i}=1$ one has $\overrightarrow{v_{i}}=-\vec{v}_{i}^{0}$, i.e., a pedestrian moves backward with desired velocity. Even values of $s_{i}$ higher than 1 are not excluded and can occur during a simulation. Therefore, a careful calibration of $\eta$ is required to achieve an optimal balance between overlapping and oscillations.

Unfortunately, it is not possible to adjust the strength of the repulsive force by means of $\eta$ in order to get an overlapping-free and meanwhile an oscillation-free simulation. Nevertheless, by proper choice of $\eta$ one can reduce the amount of overlapping among pedestrians such that it becomes negligible and can be interpreted as a deformation. This characteristic of the GCFM is not fulfilled by the CFM [5], where total overlapping $\left(o_{i j}=1\right)$ can be observed.

Furthermore, the quantities $o^{(s)}$ and $o^{(v)}$ provide a criterion to choose an optimal value for $\eta$, which is given by the intersection of the curves representing $o^{(s)}$ and $o^{(v)}$.

\section{HARD CIRCLES VS DYNAMICAL CIRCLES: THE FUNDAMENTAL DIAGRAM FOR SINGLE FILE MOVEMENT}

It is suggested that the effective space requirement of a moving pedestrian varies with velocity. Usually, the projec- tion of the pedestrian's shape to the two-dimensional plane is modeled as a circle with a radius $r[3,7,10]$. Thompson suggested a three-circle representation for main body and shoulders [22]. According to [23], however, the radius of the circle varies such that the space requirement of pedestrians increases significantly as speed increases. In [14] a linear velocity dependence

$$
r_{i}=r_{\min }+\tau_{r} v_{i}
$$

of the radius with parameters $r_{\min }$ and $\tau_{r}$ was suggested. "Space requirement" encompasses the physical area taken by the torso together with the motion of the legs, lateral swaying, and a safety margin.

The repulsive force reads

$$
F_{i j}^{\overrightarrow{\mathrm{eep}}}=-m_{i} k_{i j} \frac{\left(\eta v_{i}^{0}+v_{i j}\right)^{2}}{\mathrm{~d}_{i j}} \overrightarrow{e_{i j}},
$$

with

$$
\mathrm{d}_{i j}=R_{i j}-r_{i}\left(v_{i}\right)-r_{j}\left(v_{j}\right)
$$

the effective distance between pedestrian $i$ and $j$ and $r_{i}$ the radius of pedestrian $i$ as defined in Eq. (18).

\section{ELLIPTICAL VOLUME EXCLUSION OF PEDESTRIANS}

One drawback of circles that impact negatively the dynamics is their rotational symmetry with respect to their centers. Therefore, they occupy the same amount of space in all directions. In single file movement this is irrelevant since the circles are projected to lines and only the required space in movement direction matters. However, in two-dimensional movement the aforementioned symmetry lasts by occupying unnecessary lateral space.

In [24], Fruin introduced the "body ellipse" to describe the plane view of the average adult male human body. Pauls [23] presented ideas about an extension of Fruin's ellipse model to better understand and model pedestrian movement as density increases. Templer [25] noticed that the so called "sensory zone," which is a bubble of space between pedestrians and other objects in the environment to avoid physical conflicts and for psychocultural reasons, varies in size and takes the shape of an ellipse. In fact, ellipses are closer to the projection of required space of the human body on the plane, including the extent of the legs during motion and the lateral swaying of the body.

Having the ambition to describe with the same set of parameters the dynamics in one- and two-dimensional space we extend our model by introducing an elliptical volume exclusion of pedestrians. Given a pedestrian $i$ we define an ellipse with center $\left(x_{i}, y_{i}\right)$, major semiaxis $a$ and minor semiaxis $b$. $a$ models the space requirement in the direction of movement. In analogy to Eq. (18) we set

$$
a=a_{\min }+\tau_{a} v_{i}
$$

with two parameters $a_{\min }$ and $\tau_{a}$.

Fruin [24] observed body swaying during both human locomotion and while standing. Pauls [26] remarks that swaying laterally should be considered while determining the re- 

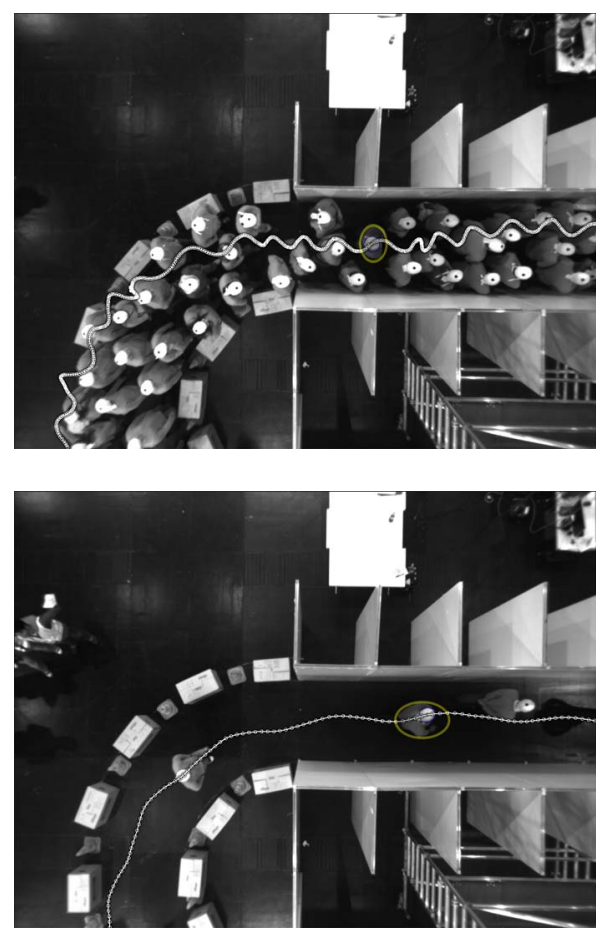

FIG. 5. (Color online) Off-line trajectory detection with PeTrack [27]. Top: the trajectory of the detected pedestrian shows strong swaying. Bottom: the faster pedestrians move, the smoother and weaker is the swaying of their trajectories.

quired width of exit stairways. In [20] characteristics of lateral swaying are determined experimentally. Observations of experimental trajectories in [20] indicate that the amplitude of lateral swaying varies from a maximum $b_{\max }$ for slow movement and gradually decreases to a minimum $b_{\min }$ for free movement when pedestrians move with their free velocity (Fig. 5). Thus we describe with $b$ the lateral swaying of pedestrians and set

$$
b=b_{\max }-\left(b_{\max }-b_{\min }\right) \frac{v_{i}}{v_{i}^{0}} .
$$

Since $a$ and $b$ are velocity dependent, the inequality

$$
b \leq a
$$

does not always hold for the ellipse $i$. In the rest of this work we denote the semi-axis in the movement direction by $a$ and its orthogonal semi-axis by $b$.

\section{ELLIPTICAL VOLUME EXCLUSION AND FORCE IMPLEMENTATION}

In this section we give some mathematical insights concerning the implementation of the repulsive forces.

\section{A. Repulsive forces between pedestrians}

In order to calculate the repulsive force emerging from pedestrian $j$ acting on pedestrian $i$ according to Eq. (19) we require the distance between the borders of the ellipses,

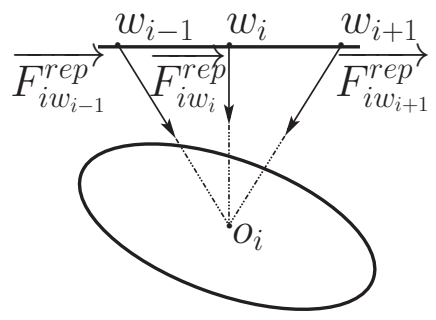

FIG. 6. Each wall is modeled as three static point masses acting on pedestrians.

along a line connecting the two pedestrians $\mathrm{d}_{i j}$. See Appendix A for more details on $\mathrm{d}_{i j}$.

Another important quantity is the distance of closest approach or contact distance of two ellipses $\tilde{l}$ which is the minimum of $\mathrm{d}_{i j}$ while $i$ and $j$ are not overlapping. Unlike for circles, $\tilde{l}$ can be nonzero for ellipses and depends on their orientations. In [28] an analytical expression for the distance of the closest approach of two ellipses with arbitrary orientation is derived. Figure 7 shows how $\mathrm{d}_{i j}$ and $\tilde{l}$ goes in the repulsive force.

\section{B. Repulsive forces between pedestrians and walls}

The repulsive force between a pedestrian $i$ and a wall is zero if $i$ performs a parallel motion to the wall. While this behavior of the force is correct, it leads to very small repulsive forces when the pedestrians' motion is almost parallel to the wall. For this reason we characterize in this model walls by three point masses acting on pedestrians within a certain interaction range (Fig. 6). The middle point is the point with the shortest distance from the center of the pedestrian to the line segment of the wall. All three points have to be computed at each step as the pedestrian moves. The distance between the three wall points is set to the minor semi-axis of an ellipse. If one lateral point $\left(w_{i+1}\right.$ or $\left.w_{i+1}\right)$ does not lie on the line segment of the wall, then it will not be considered in the computation of the repulsive force.

The number of point masses has been chosen by a process of trial and error. Simulations have shown that three point masses are sufficient to keep pedestrians away from walls. Meanwhile they are computationally cost-effective.

As walls are static objects, the repulsive force emerging from a wall $w$ and acting on pedestrian $i$ simplifies to

$$
F_{i w}^{\overrightarrow{\mathrm{rep}}}=\sum_{j=i-1}^{i+1} F_{i w_{j}}^{\overrightarrow{\mathrm{rep}}}
$$

with

$$
F_{i w_{j}}^{\overrightarrow{\mathrm{rep}}}=-m_{i} k_{i w_{i}} \frac{\left(\eta v_{i}^{0}+v_{i}^{n}\right)^{2}}{\mathrm{~d}_{i w_{j}}} e_{i w_{j}}, \quad j \in\{i-1, i, i+1\} .
$$

$v_{i}^{n}$ is the component of the velocity normal to the wall, $k_{i w_{i}}$ and $\vec{e}_{i w_{j}}$ as defined, respectively, in Eqs. (8) and (5) in Sec. II.

The distance between a line $w$ and the ellipse $i$ is 


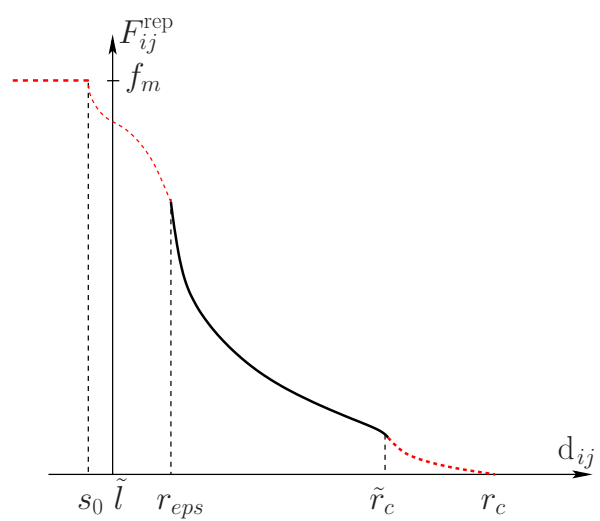

FIG. 7. (Color online) The interpolation of the repulsive force between pedestrians $i$ and $j$ Eq. (19) depending on $\mathrm{d}_{i j}$ and the distance of closest approach $\tilde{l}$, see Sec. VI A. As the repulsive force also depends on the relative velocity $v_{i j}$, this figure depicts the curve of the force for $v_{i j}=$ const. The left and right dashed curves are defined in Eqs. (28) and (27) respectively. The wall-pedestrian interaction has an analogous form with $\mathrm{d}_{i j}$ and $\tilde{l}$ replaced by $\mathrm{d}_{w i}$ and $\tilde{k}$, respectively.

$$
\mathrm{d}_{i w}=k_{i}-r_{i},
$$

with $r_{i}$ the polar radius determined in Eq. (A3) and $k_{i}$ the distance of point $o_{i}$ to the line $w$. Further details can be found in Appendix B. According to the distance $\tilde{l}$ defined for the repulsive forces between pedestrian in Sec. VI A, we introduce the distance of the closest approach between an ellipse and a line $\tilde{k}$, see Appendix B and Fig. 7 for details.

Note that in Eq. (25), $k_{i w_{i}}$ in the force is independent of the chosen lateral wall point $w_{j}$. That means, if a pedestrian is moving parallel to the wall, $k_{i w_{i}}=0$ and thus the points $j-1$ and $j+1$ have no effects.

\section{Numerical stabilization of the repulsive force}

In this section, we describe a numerical treatment of the repulsive force. For the sake of simplicity, we focus on the case of pedestrian-pedestrian interactions. The pedestrianwall case is treated similarly.

The strength of the repulsive force decreases with increasing distance between two pedestrians. Nevertheless the range of the repulsive force is infinite. This is unrealistic for interactions between pedestrians. Therefore, we introduce a cutoff radius $r_{c}=2 \mathrm{~m}$ for the force limiting the interactions to adjacent pedestrians solely. To guarantee robust numerical integration a two-sided Hermite-interpolation of the repulsive force is implemented. The interpolation guarantees that the norm of the repulsive force decreases smoothly to zero for $\mathrm{d}_{i j} \rightarrow r_{c}^{-}$. For $\mathrm{d}_{i j} \rightarrow \widetilde{l}^{+}$the interpolation avoids an increase of the force to infinity but to $f_{m}=3 F_{i j}^{\mathrm{rep}}\left(r_{\mathrm{eps}}\right)$ at $s_{0}=r_{\mathrm{eps}}$ and $r_{\text {eps }}=0.1 \mathrm{~m}$, where it remains constant. $\mathrm{d}_{i j}$ and $\tilde{l}$ are illustrated in Sec. VI A. Figure 7 shows the dependence of the repulsive force on the distance for constant relative velocity.

The right interpolation function $P_{r}$ and the left one $P_{l}$ (dashed parts of the function in Fig. 7) are defined using

$$
\begin{gathered}
P_{r}\left(\widetilde{r}_{c}\right)=F_{i j}^{\mathrm{rep}}\left(\widetilde{r}_{c}\right), \quad P_{r}\left(r_{c}\right)=0, \\
\left(P_{r}\right)^{\prime}\left(\widetilde{r}_{c}\right)=\left(F_{i j}^{\mathrm{rep}}\right)^{\prime}\left(\widetilde{r}_{c}\right),\left(P_{r}\right)^{\prime}\left(r_{c}\right)=0,
\end{gathered}
$$

with $\widetilde{r}_{c}=r_{c}-r_{\text {eps }}$ and

$$
\begin{gathered}
P_{l}\left(s_{0}\right)=f_{m}, \quad P_{l}\left(r_{\mathrm{eps}}\right)=F_{i j}^{\mathrm{rep}}\left(r_{\mathrm{eps}}\right), \\
\left(P_{l}\right)^{\prime}\left(s_{0}^{+}\right)=1, \quad\left(P_{l}\right)^{\prime}\left(r_{\mathrm{eps}}\right)=\left(F_{i j}^{\mathrm{rep}}\right)^{\prime}\left(r_{\mathrm{eps}}\right) .
\end{gathered}
$$

where the prime indicates the derivative. $s_{0}$ is the minimum allowed magnitude of the effective distance of two ellipses. Due to the superposition of the forces the inequality,

$$
\mathrm{d}_{i j} \geq s_{0} .
$$

for pedestrians $i$ and $j$ is not guaranteed.

\section{SIMULATION RESULTS}

The initial value problem in Eq. (3) was solved using an Euler scheme with fixed-step size $\Delta t=0.01 \mathrm{~s}$. First the state variables of all pedestrians are determined. Then the update to the next step is performed. Thus, the update in each step is parallel.

The desired speeds of pedestrians are Gaussian distributed with mean $\mu=1.34 \mathrm{~m} / \mathrm{s}$ and standard deviation $\sigma=0.26 \mathrm{~m} / \mathrm{s}$. The time constant $\tau$ in the driving force Eq. (4) is set to $0.5 \mathrm{~s}$, i.e., $\tau \gg \Delta t$. For simplicity, the mass $m_{i}$ is set to unity. In all simulations we set $\eta$ [Eqs. (19) and (25)] to 0.2 .

In order to verify the model and evaluate the difference of the elliptical shape of the volume exclusion versus the circular one we measure the fundamental diagram in twodimensional space with the same set of parameter as for the one-dimensional fundamental diagram. In the onedimensional case only the space requirement of pedestrians in movement direction, expressed in terms of the semiaxis $a$, influences the dynamics of the system. We set $a_{\min }$ $=0.18 \mathrm{~m}$ and $\tau_{a}=0.53 \mathrm{~s}$ [see Eq. (21)].

To illustrate the impact of the velocity dependence of the radius on the dynamics of pedestrians we measure the onedimensional fundamental diagram in a corridor of $26 \mathrm{~m}$ with periodic boundary conditions. The measurement segment is 2 $\mathrm{m}$ long and situated in the middle of the corridor. Details about the measurement method are given in Appendix C.

The results for the one-dimensional fundamental diagram are shown in Fig. 8 and compare well with experimental data. Ellipses with velocity-dependent semiaxes emulate the space requirement of the projected shape of pedestrians better. Even the shape of the fundamental diagram is reproduced after inclusion of this velocity dependence.

We extend the simulation to two-dimensional space and simulate a $25 \times 1 \mathrm{~m}^{2}$ corridor with periodic boundary conditions. A measurement segment of $2 \times 1 \mathrm{~m}^{2}$ was set in the middle of the corridor. We use the same measurement method as for the single-file case (see Appendix C). Calibration of the parameters of the lateral semi-axis $b\left[b_{\min }\right.$ and $b_{\max }$ in Eq. (22)] leads to the values $b_{\min }=0.2 \mathrm{~m}$ and $b_{\max }=0.25 \mathrm{~m}$. The simulation result is shown in Fig. 9 . 

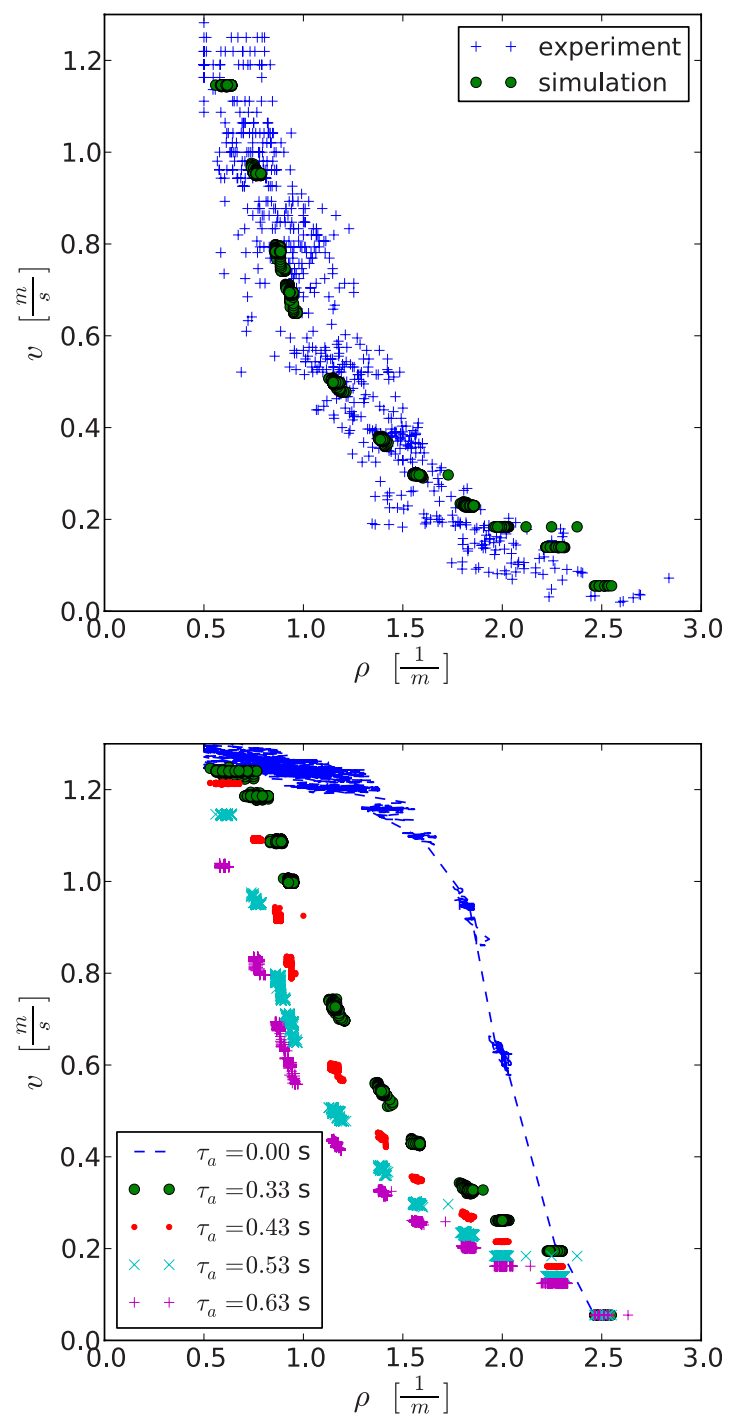

FIG. 8. (Color online) Top: Velocity-density relation for onedimensional movement compared to experimental data [29]. For the simulations, $\tau_{a}$ is set to $0.53 \mathrm{~s}$. Bottom: changing $\tau_{a}$ in Eq. (21) influences the slope of the diagram. $a_{\min }$ has been kept equal to 0.18 m. $\tau_{a}=0$ represents pedestrians with constant space requirement.

With the chosen dimensions of the semiaxes $a$ and $b$ the model yields the right relation between velocity and density both in single-file movement and wide corridors, although only a corridor width of $1 \mathrm{~m}$ was investigated. One remarks that the fundamental diagram for elliptical shaped particles is an upper bound for that of circular ones, especially at low and medium densities. At high densities there is no noticeable difference between both shapes.

\section{CONCLUSIONS}

We have proposed modifications of a spatially continuous force-based model [5] to describe quantitatively the movement of pedestrians in one- and two-dimensional space. Besides being a remedy for numerical instabilities in CFM the modifications simplify the approach of Yu et al. [5] since we can dispense with their extra "collision detection technique"

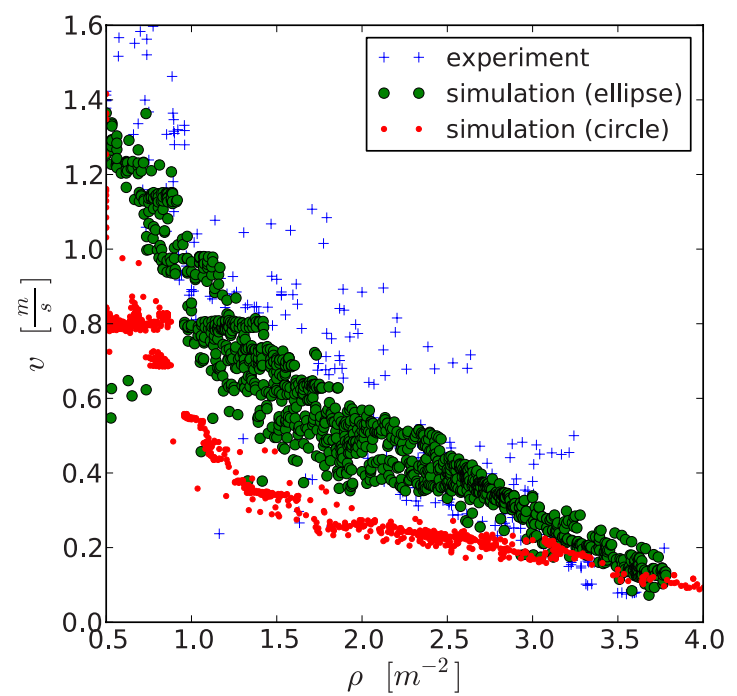

FIG. 9. (Color online) Density-velocity relation in a corridor of dimensions $25 \times 1 \mathrm{~m}^{2}$ in comparison with experimental data obtained in the HERMES-project [30]. For the simulation with circles, $b$ is set to be equal to $a$.

without deteriorating performance. The implementation of the model is straightforward [32,33] and does not use any restrictions on the velocity. Furthermore, we introduced an elliptical volume exclusion of pedestrians and studied its influence compared to the standard circular one. Simulation results show good agreement with experimental data. Nevertheless, the model contains free parameters that have to be tuned adequately to adapt the model to a given scenario. Further improvement of the model could be made by including, for example, a density-dependent repulsive force.

Although the model describes quantitatively well the operative level of human behavior, it does not consider aspects of the tactical and strategic levels [31]. Phenomena such as cooperation, changing lanes, and overtaking are not reproduced, especially in bidirectional flow.

\section{ACKNOWLEDGMENTS}

The authors are grateful to the Deutsche Forschungsgemeinschaft (DFG) for funding this project under Grant No. SE 1789/1-1.

\section{APPENDIX A: DISTANCE BETWEEN TWO ELLIPSES}

In this appendix we give details about the calculation of the distance $d_{i j}$ between two ellipses which is defined as the distance between the borders of the ellipses, along a line connecting their centers (Fig. 10).

By proper choice of the coordinate system the ellipse $i$ may be written as quadratic form,

$$
\frac{x^{2}}{a_{i}^{2}}+\frac{y^{2}}{b_{i}^{2}}=1 .
$$

In polar coordinates, with the origin at the center of the ellipse and with the angular coordinate $\alpha_{i}$ measured from the major axis, one gets 


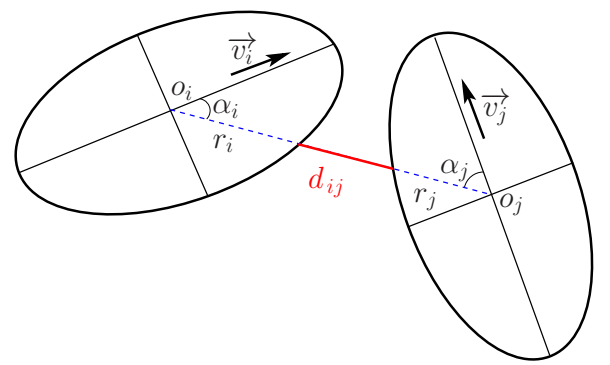

FIG. 10. (Color online) $\mathrm{d}_{i j}$ is the distance between the borders of the ellipses $i$ and $j$ along a line connecting their centers.

$$
x=r_{i} \cos \left(\alpha_{i}\right), \quad y=r_{i} \sin \left(\alpha_{i}\right) .
$$

By replacing the expressions of $x$ and $y$ in Eq. (A1) and rearranging we obtain the expression

$$
q r_{i}^{2}-1=0
$$

for the polar radius $r_{i}$ with

$$
q=\frac{\cos ^{2} \alpha_{i}}{a_{i}^{2}}+\frac{\sin ^{2} \alpha_{i}}{b_{i}^{2}} .
$$

In the same manner, we determine the polar radius $r_{j}$.

Finally, the distance $\mathrm{d}_{i j}$ between the centers of the ellipses $i$ and $j$ is determined as follows (Fig. 10),

$$
\mathrm{d}_{i j}=\left\|\vec{o}_{i} o_{j}\right\|-r_{i}-r_{j} .
$$

Note that the distance between two ellipses can be nonzero even when the ellipses touch or overlap.

\section{APPENDIX B: DISTANCE OF CLOSEST APPROACH}

Distance of closest approach of two ellipses is the smallest distance between their borders, along a line connecting their centers while they are not overlapping. See Fig. 11, top. To mitigate overlapping the repulsive forces are high for distances in a certain neighborhood of the distance of closest approach, see $\tilde{l}$ in Fig. 7. An analytical solution of this dis-
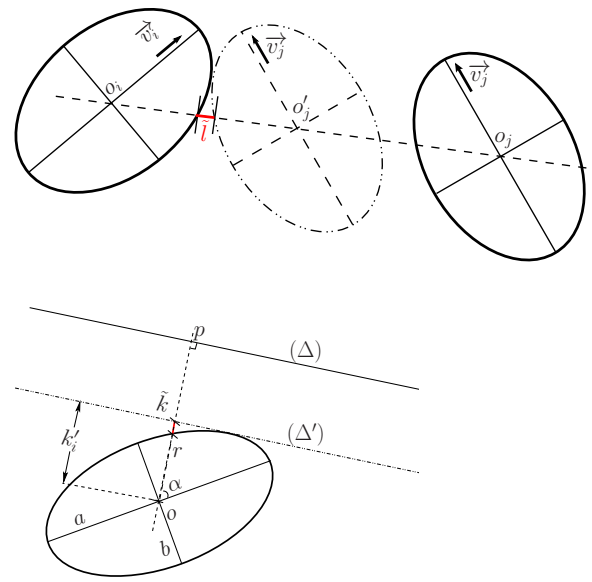

FIG. 11. (Color online) Top: distance of closest approach of two ellipses. Bottom: distance of closest approach between an ellipse and a line. tance for two arbitrary ellipses is presented in [28].

In this appendix we describe an algorithm to calculate the distance of closest approach of an ellipse and a line $(\Delta)$, which is the distance between the border of the ellipse, along a line connecting its center $o$ and the closest point on the line to $o$. For this purpose consider without loss of generality an ellipse $i$ in canonical position and let $\left(\Delta^{\prime}\right)$ be the line tangential to the ellipse $i$ and parallel to $(\Delta)$ (Fig. 11, bottom),

$$
(\Delta): y=c x+d, \quad\left(\Delta^{\prime}\right): y=c x+d^{\prime} .
$$

with known coefficients $c$ and $d$.

To determine $d^{\prime}$ we solve the intersection equations of an ellipse and a line, which yields the quadratic equation

$$
q^{\prime} x^{2}+p^{\prime} x+s^{\prime}=0
$$

with

$$
q^{\prime}=\frac{1}{a^{2}}+\frac{c^{2}}{b^{2}}, \quad p^{\prime}=\frac{2 c d^{\prime}}{b^{2}} \quad \text { and } \quad s^{\prime}=\frac{d^{\prime 2}}{b^{2}}-1
$$

As $\left(\Delta^{\prime}\right)$ is tangential to the ellipse we have

$$
D=0
$$

with $D$ the discriminant of Eq. (B2). Solving Eq. (B4) gives

$$
d^{\prime}= \pm \sqrt{b^{2}+a^{2} c^{2}} \text {. }
$$

Finally the distance of closest approach of the ellipse $i$ and line $(\Delta)$ is

$$
\tilde{k}=k_{i}^{\prime}-r_{i}
$$

with $k_{i}^{\prime}$ the distance of $c_{i}$ to $\left(\Delta^{\prime}\right)$ and $r_{i}$ the polar radius as determined in Eq. (A3).

\section{APPENDIX C: MEASUREMENT METHOD}

The mean velocity of pedestrian $i$ that enters the measurement are at $\left(x_{i}^{\text {in }}, y_{i}^{\text {in }}\right)$ and leaves it at $\left(x_{i}^{\text {out }}, y_{i}^{\text {out }}\right)$ is determined as

$$
v_{i}=\frac{\sqrt{\left(x_{i}^{\text {out }}-x_{i}^{\text {in }}\right)^{2}+\left(y_{i}^{\text {out }}-y_{i}^{\text {in }}\right)^{2}}}{t_{i}^{\text {out }}-t_{i}^{\text {in }}},
$$

where $t_{i}^{\text {in }}$ is the entrance time and $t_{i}^{\text {out }}$ exit time of $i$. For the one-dimensional case $y_{i}^{\text {in }}=y_{i}^{\text {out }}=0$.

The density is defined as follows:

$$
\begin{gathered}
\rho_{i}=\frac{1}{t_{i}^{\text {out }}-t_{i}^{\text {in }}} \int_{t_{\text {in }}}^{t_{\text {out }}} \rho(t) d t \\
\rho(t)=\frac{N_{\text {in }}(t)}{l_{m}} .
\end{gathered}
$$

with $l_{m}=2 m$ the length of the measurement area in the movement direction and $N_{\text {in }}(t)$ is the number of pedestrians within the area at time $t$. In one-dimensional space the measurement area is reduced to a measurement segment of length $l_{m}$. 
[1] A. Schadschneider, W. Klingsch, H. Klüpfel, T. Kretz, C. Rogsch, and A. Seyfried, in Encyclopedia of Complexity and System Science, edited by R. A. Meyers (Springer, Berlin, Heidelberg, 2009), pp. 3142-3176.

[2] A. Schadschneider, D. Chowdhury, and K. Nishinari, Stochastic Transport in Complex Systems: From Molecules to Vehicles (Elsevier, New York, 2010).

[3] D. Helbing and P. Molnár, Phys. Rev. E 51, 4282 (1995).

[4] D. Helbing, Comput. Mater. Sci. 30, 180 (2004).

[5] W. J. Yu, R. Chen, L. Y. Dong, and S. Q. Dai, Phys. Rev. E 72, 026112 (2005).

[6] T. I. Lakoba, D. J. Kaup, and N. M. Finkelstein, Simulation 81, 339 (2005).

[7] D. R. Parisi and C. O. Dorso, Physica A 385, 343 (2007).

[8] R. Löhner, Appl. Math. Model. 34, 366 (2010).

[9] S. P. Hoogendoorn, W. Daamen, and R. Landman, in Pedestrian and Evacuation Dynamics 2005 (Springer, New York, 2007), p. 253.

[10] A. Johansson, D. Helbing, and P. K. Shukla, Adv. Complex Syst. 10, 271 (2007).

[11] T. Kretz, S. Hengst, and P. Vortisch, Pedestrian Flow at Bottlenecks-Validation and Calibration of Vissim's Social Force Model of Pedestrian Traffic and its Empirical Foundations, edited by M. Sarvi,International Symposium of Transport Simulation 2008 (ISTS08), Gold Coast, Australia (Monash University, Victoria, Australia 2008).

[12] D. R. Parisi, M. Gilman, and H. Moldovan, Physica A 388, 3600 (2009).

[13] S. P. Hoogendoorn and W. Daamen, in Pedestrian Behavior, edited by H. Timmermans (Emerald, Bingley, UK, 2009), p. 195.

[14] A. Seyfried, B. Steffen, and T. Lippert, Physica A 368, 232 (2006).

[15] A. Kirchner and A. Schadschneider, Physica A 312, 260 (2002).

[16] A. Kirchner, K. Nishinari, and A. Schadschneider, Phys. Rev. E 67, 056122 (2003).

[17] A. Kirchner, H. Klüpfel, K. Nishinari, A. Schadschneider, and M. Schreckenberg, Physica A 324, 689 (2003).

[18] D. Yanagisawa, A. Kimura, A. Tomoeda, N. Ryosuke, Y. Suma, K. Ohtsuka, and K. Nishinari, Phys. Rev. E 80, 036110 (2009).
[19] S. P. Hoogendoorn, W. Daamen, and P. H. L. Bovy, in Transportation Research Board Annual Meeting (National Academy Press, Washington, DC, 2003), pp. 1-15.

[20] S. P. Hoogendoorn and W. Daamen, Transp. Sci. 39, 147 (2005).

[21] M. Chraibi, A. Seyfried, A. Schadschneider, and W. Mackens, Quantitative Description of Pedestrian Dynamics with a Force-based Model, in 2009 IEEE/WIC/ACM International Joint Conference on Web Intelligence and Intelligent Agent Technology (IEEE Computer Society, Los Alamitos, CA, 2009), Vol. 3, pp. 583-586.

[22] P. A. Thompson and E. W. Marchant, Fire Saf. J. 24, 131 (1995).

[23] J. Pauls, Suggestions on evacuation models and research questions. Human Behaviour in Fire, Proceedings of the Third International Symposium on Human Behaviour in Fire, Ulster, Belfast, edited by T. J. Shields (Interscience, London, 2004).

[24] J. J. Fruin, Pedestrian Planning and Design (Elevator World, New York, 1971).

[25] J. A. Templer, The Staircase: Studies of Hazards, Falls, and Safer Design (The MIT Press, Cambridge, MA, 1992).

[26] J. Pauls, Stairways and Ergonomics, 2006, Proceedings of American Society of Safety Engineers Annual Professional Development Conference, Seattle, 2006 (ASSE, IL, 2006).

[27] M. Boltes, A. Seyfried, B. Steffen, and A. Schadschneider, in Pedestrian and Evacuation Dynamics 2008 (Springer, New York, 2010), p. 43.

[28] X. Zheng and P. Palffy-Muhoray, Phys. Rev. E 75, 061709 (2007).

[29] A. Seyfried, M. Boltes, J. Kähler, W. Klingsch, A. Portz, T. Rupprecht, A. Schadschneider, B. Steffen, and A. Winkens, in Pedestrian and Evacuation Dynamics 2008 (Springer, 2010), p. 145.

[30] S. Holl and A. Seyfried, inSiDe 7, 60, 2009.

[31] A. Schadschneider, H. Klüpfel, T. Kretz, and A. Rogsch, in Multi-Agent Systems for Traffic and Transportation Engineering, edited by A. Bazzan and F. Klügl (IGI Global, Hershey, Pennsylvania, 2009), Chap. 6, pp. 124-154.

[32] GNU General public license http://www.gnu.org/licenses/ gpl.html

[33] http://www.fz-juelich.de/jsc/ped 